\title{
Broadband Information Broadcasting using LED-based Interior Lighting
}

\author{
Jelena Grubor, Sebastian Randel, Klaus-Dieter Langer, and Joachim W. Walewski
}

\begin{abstract}
Emergence of white-light LEDs allows the combination of lighting and information broadcast functionality in one optical source. We investigate analytically and by Monte Carlo simulations feasible data transmission rates in a moderatesize office room, where we assume illumination conforming to standards and the use of commercially available LEDs and photodiodes. The performances of systems relying on base-band (i.e. Pulse-Amplitude Modulation -PAM) and discrete multi-tone (DMT) transmission show that data rates of more than $100 \mathrm{Mbit} / \mathrm{s}$ can be expected despite the rather low bandwidth of the system.
\end{abstract}

Index Terms-Discrete multi-tone, Optical wireless, Visible light communication, White-light LED.

\section{INTRODUCTION}

$\mathrm{O}$ PTICAL wireless technology (OW) presents a promising supplement to already existing wireless radio-frequency (RF) technologies like e.g. WLAN and Bluetooth. It offers broad, worldwide available and unlicensed bandwidth, which does not interfere with radio bands. Hence, this emerging technology is very attractive for RF-sensitive operating environments. A special case of $\mathrm{OW}$ is communication via visible light, where an additional advantage lies in synergetic potential for simultaneous use of light sources for lighting and communication.

The idea of bringing these two worlds together was recently re-invigorated through the emergence of high-power white-light LEDs, which offer a considerable modulation bandwidth $(\sim 2-20 \mathrm{MHz}),[1]$, [2]. Moreover, they posses clear advantages over conventional lighting sources (such as a potentially high power efficiency and long life-time expectancy), which makes them a strong candidate for future illumination scenarios. With visible-light wireless systems, it would be possible to broadcast broadband information wherever general lighting is used, e.g., in offices or public and private transport. One could also imagine transmission using

Manuscript received February, 28, 2008. This contribution was partially published at the German national conference "Breitbandversorgung in Deutschland - Vielfalt für alle?”, ITG Fachbericht 198, Berlin, 2007.

J. Grubor and K.-D. Langer are with the Fraunhofer Institute for Telecommunications, Heinrich-Hertz-Institut, Einsteinufer 37, DE-10587 Berlin, Germany (phone: +49-30-31002-634; fax: 49-30-31002-250; e-mail: grubor@hhi.fraunhofer.de).

S. Randel and J. W. Walewski are with the Siemens Corporate Technology, Information and Communications, Networks and Multimedia Communications, Otto-Hahn-Ring 6, DE-81739 Munich, Germany illuminated advertising [6], communication between cars (using their rear and front lights), between traffic infrastructure and cars [7], [8], or between machines (robots). Moreover, visible light communication may be part of a system to provide broadband internet and mobile services even on board of aircrafts.

Several reported investigations for office application relied on complex tri-chromatic LEDs with idealized bandwidths of $100 \mathrm{MHz}$ and more, [3]-[5]. In our study, we revisit the work of Komine et. al., [4], but consider commercially available "single-chip" white-light LEDs (blue LED plus phosphorous layer). By theoretical analysis, we investigate the potentials for high-speed transmission in a moderate-size office, with both base-band and discrete multi-tone (DMT) modulation. The use of DMT signals was previously considered in [9], [10], however in combination with very low bit rates.

This paper is organized as follows. Section II provides the framework for investigation, including an overview on the relevant characteristics of LED chips as well as a scenario for the chip layout in order to achieve the target lighting levels. Within Section III, the communication channel is introduced and discussed in terms of inter-symbol interference, after which the system performance and achievable transmission rates are investigated analytically and by simulations of baseband (i.e. pulse-amplitude modulation, PAM) and DMT-based transmission. Finally, results of our investigations are summarized and major conclusions are drawn in Section IV.

\section{ILLUMinATION With WhitE-LIGHT LEDS}

\section{A. Framework for Investigation}

In order to make the results of our investigation comparable to those used in the pioneer study of Komine et al. [4], we consider an empty model room with identical dimensions (see Fig. 1).

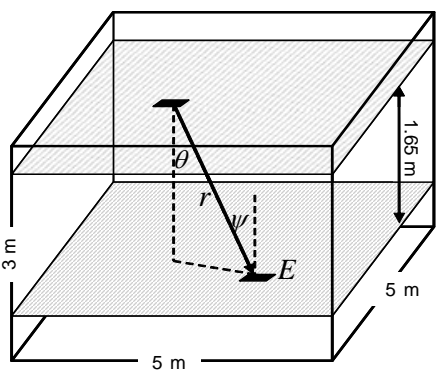

Fig. 1: Model room. Definition of the shown parameters will be introduced in section II B. 
The room is a $5 \times 5 \times 3 \mathrm{~m}^{3}$ cube, and the optical sources are installed $2.5 \mathrm{~m}$ above the floor. The room is assumed to serve as office, and the main purpose of the lighting is to illuminate the writing desk. Therefore, we evaluate the system performance at desk-top height $(0.85 \mathrm{~m}$ above the floor).

For proper lighting, a certain brightness of the illuminated surface is required, and for a reliable high-speed data transmission, sufficient optical power is needed. Both of these conditions need to be considered in the system design.

\section{B. LED Chip Characteristics}

Even though white light can be produced by proper mixing of red, green and blue light ( 3 chips), most of the present whitelight LEDs are based on a blue LED chip topped with a phosphor layer. The power spectrum of such an LED, [11], was measured with an optical spectrum analyser (Yokogawa, AQ6315A), and the inferred spectrum is displayed in Fig. 2.

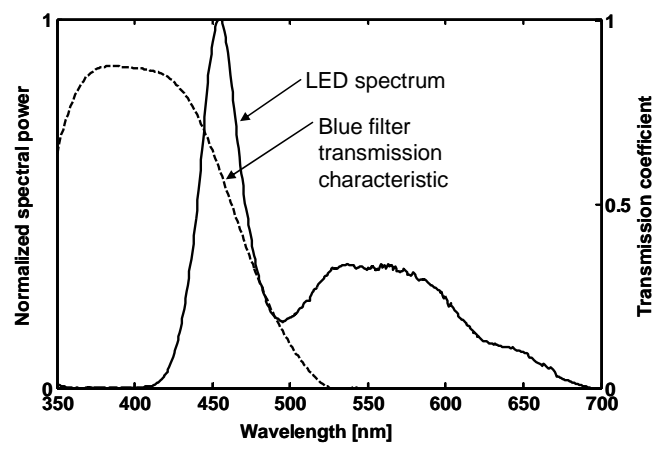

Fig. 2: Measured radiation spectrum of a phosphorescent white-light LED [11] together with the transmission characteristic of the used blue filter.

From radiation spectrum $p(\lambda)[\mathrm{W} / \mathrm{m}]$, one can obtain the luminous flux, $\Phi[\mathrm{lm}]$, of the source by evaluating

$$
\Phi=683 \frac{\operatorname{lm}}{W_{380 \mathrm{~nm}}^{720 \mathrm{~nm}}} \int p(\lambda) V(\lambda) \mathrm{d} \lambda,
$$

where $V(\lambda)$ denotes the eye sensitivity function [12]. The luminous flux is the optical power of the source as perceived by the human eye. The parameter of interest for us is the illuminance, $E[\mathrm{~lx}]$, which expresses the brightness of the illuminated surface. According to [12], it is defined as luminous flux per unit area

$$
E=\partial \Phi / \partial A=I(\theta) / r^{2}
$$

and depends on the source luminous intensity, $I(\theta)$ [cd], in the direction $\theta$. E accounts for the radiation pattern of the light source and the distance to the illuminated surface, $r$ (see Fig. 1). For a source with Lambert radiation characteristic and an angle of incidence $\psi$ [rad], the horizontal illuminance is

$$
E_{h}=I_{0} \cos ^{m}(\theta) \cos \psi / r^{2}
$$

where $I_{0}=I(\theta=0)=(m+1) \Phi /(2 \pi)$ is the maximal luminous intensity [12]. The Lambert index $m$ depends on the source radiation semi-angle $\theta_{\max }$ as

$$
m=-1 / \log _{2}\left(\cos \theta_{\max }\right) .
$$

Relevant optical source parameters for data transmission are its radiant power and modulation bandwidth. The source optical power, $P[\mathrm{~W}]$ (radiant flux), is given as

$$
P=\int p(\lambda) \mathrm{d} \lambda
$$

Note, that there is no relationship to the eye sensitivity.

With the knowledge of $p(\lambda)$ and $V(\lambda)$, both $P$ and $\Phi$ were evaluated and a conversion factor of $4.2 \mathrm{~mW} / \mathrm{lm}$ for this type of LEDs was obtained. Figure 3 shows the measured modulation bandwidth of the LED [11].

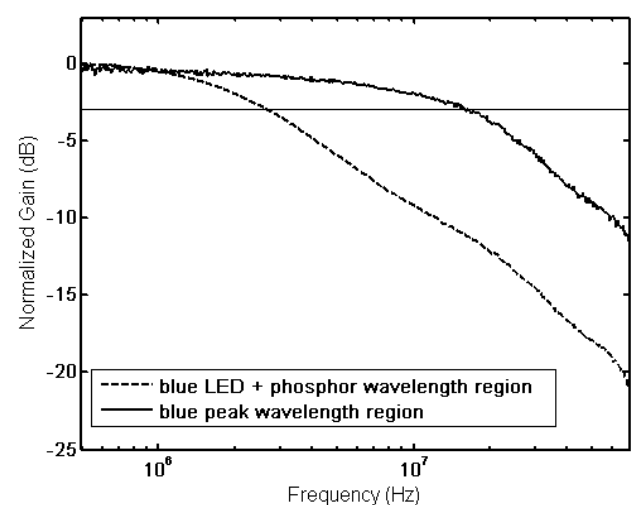

Fig. 3: Measured modulation spectrum of a phosphorescent white-light LED [11]. The gain represents the electrical gain of the detector. The solid horizontal line tags the $3-\mathrm{dB}$ modulation bandwidth.

This measurement has been carried out by supplying the DC driving voltage from a current source and the AC voltage from a network analyser. The emitted light was detected with a preamplified photodiode and the diode output was then monitored with the a network analyser. From this figure one can see that, by suppressing the slow phosphorescent portion of the optical spectrum $(\sim 500-700 \mathrm{~nm})$, the modulation bandwidth can be enhanced from 2 to $\sim 20 \mathrm{MHz}$. In order to attain high modulation bandwidths and thus high data rates, only the blue part of the spectrum should be detected (see Fig. 2). About $50 \%$ of the optical power resides in this spectral region, so that the conversion factor for the optical power available in the blue peak region (for a source with luminous flux $\Phi$ ) is $2.1 \mathrm{~mW} / \mathrm{lm}$ at maximum.

Although bandwidth measurement and conversion-factor calculation were done for a particular LED, it is reasonable to use the obtained values for other chips manufactured in a similar way. 


\section{Fulfilling the Lighting Function}

Since the general lighting can be considered as the primary purpose for an LED source (with data transmission as its secondary function), we need to ensure sufficient horizontal brightness at the desk-top surface. In accordance with EN 12464-1, we regard $400 \mathrm{~lx}$ as a minimal brightness at the desk-top height in the area where a working place is set, and aim for a 200-800 lx span in the whole room [13].

Throughout our study, we investigated several commercially available LEDs and concluded that using a chip with a large radiation angle is of practical importance, since in this case only a moderate amount of LED chips is needed for room illumination, keeping the overall complexity of the system low. We chose an LED chip, whose characteristics are given in Table 1, and which is a good match for our lighting purposes. The figures for radiation angle and possible luminous intensity were provided by the manufacturer, and the output optical power was calculated with the conversion factor of $2.1 \mathrm{~mW} / \mathrm{lm}$, as reported in the previous section.

TABLE 1: SUMMARY OF RELEVANT PARAMETERS

\begin{tabular}{|c|c|c|}
\hline Parameter & Scenario A & Scenario B \\
\hline \multicolumn{3}{|c|}{ LED characteristics } \\
\hline $\begin{array}{l}\text { Half-intensity radiation } \\
\text { angle, } 2 \theta_{\max }\end{array}$ & \multicolumn{2}{|c|}{$120^{\circ}$} \\
\hline $\begin{array}{l}\text { Maximum luminous } \\
\text { intensity, } I_{0}\end{array}$ & \multicolumn{2}{|c|}{$9.5 \mathrm{~cd}$} \\
\hline $\begin{array}{l}\text { Source optical power, } \\
P_{C H I P}\end{array}$ & \multicolumn{2}{|c|}{$63 \mathrm{~mW}$} \\
\hline \multicolumn{3}{|c|}{ Scenario characteristics } \\
\hline $\begin{array}{l}\text { LED configuration at } \\
\text { the ceiling }\end{array}$ & $\begin{array}{l}\text { Uniformly } \\
\text { distributed }\end{array}$ & $\begin{array}{l}4 \text { lamps of } 1 \times 1 \\
\mathrm{~m}^{2}\end{array}$ \\
\hline LED chip spacing & $16 \mathrm{~cm}$ & $7 \mathrm{~cm}$ \\
\hline Total number of chips & 961 & 900 \\
\hline \multicolumn{3}{|c|}{ Brightness statistics } \\
\hline $\begin{array}{l}\text { Brightness } E \text { at desk-top } \\
(\min , \max )\end{array}$ & $(237,855) \mathrm{lx}$ & $(176,854) \mathrm{lx}$ \\
\hline $\begin{array}{l}\text { Normalized area with } \\
E \geq 400 \mathrm{~lx}\end{array}$ & $93 \%$ & $86 \%$ \\
\hline \multicolumn{3}{|c|}{ Transmission statistics } \\
\hline max delay on LOS & \multicolumn{2}{|c|}{$5.5 \mathrm{~ns}$} \\
\hline $3 \mathrm{~dB}$ channel bandwidth & \multicolumn{2}{|c|}{$\geq 90 \mathrm{MHz}$ (approximately) } \\
\hline $\begin{array}{l}\text { Received optical power } \\
\left.P_{R} \text { (min, av, max }\right) \\
\end{array}$ & \multicolumn{2}{|c|}{$(0.1,0.4,0.5) \mathrm{mW}$} \\
\hline$S N R_{0}(\min , \max )$ & $(62,73) \mathrm{dB}$ & $(60,73) \mathrm{dB}$ \\
\hline $\begin{array}{l}\text { Normalized area with } \\
S N R_{0} \geq 66.6 \mathrm{~dB}\end{array}$ & $94 \%$ & $87 \%$ \\
\hline
\end{tabular}

Two scenarios were considered, both of them based on the above-mentioned LED chip. Scenario A represents a ceiling that is uniformly covered with 16-cm-spaced LEDs, whereas in scenario B four distinct areas of $1 \times 1 \mathrm{~m}^{2}$ (referred to as lamps), contain 7-cm-spaced LEDs. In Fig. 4, the ceiling layout for the two scenarios is illustrated.

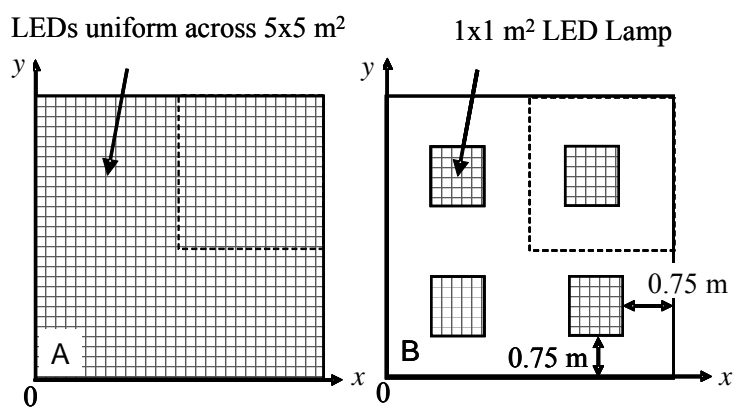

Fig. 4: Layout of ceiling lighting design for scenarios A and B (dashed areas are used for further discussions).

It is assumed that one LED chip covers a surface of $1 \mathrm{~cm}^{2}$. Both scenarios need less than 1000 chips (see Table 1). In comparison, Komine et al. considered more than 14000 chips for the very same room dimensions [4]. This large difference in the number of needed LEDs is readily explained by the more than ten times higher luminous intensity of the phosphorous LED considered here. Fig. 5 displays the distributions of the illuminance $E_{h}$ at the desk-top surface. Due to the geometric symmetry of both scenarios, only the upper right quarter of the desk-top surface (directly under the areas within dashed lines in Fig. 4) is considered in Fig. 5 (and all the following contour plots).

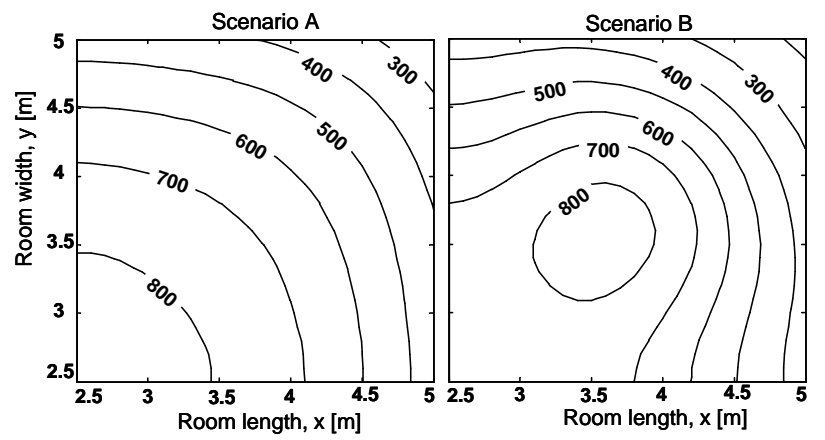

Fig. 5: Horizontal brightness distribution $E$ in $\mathrm{lx}$ at the desk-top surface for Scenarios A and B (corresponding to dashed areas in Fig.4).

In both scenarios, the aimed brightness span is met, with a major portion of the plane receiving at least $400 \mathrm{~lx}$, and thus being suitable as a work place. The exact values are given in Table 1.

\section{DAta TRANSMission With White-Light LEDS}

\section{A. Communication Channel and Inter-Symbol Interference}

The illumination at any point of the receiving surface includes lines of sight (LOS) from the LED chips as well as a contribution of reflections off the walls or objects in the room. The channel response of the LOS contributions can be modelled by Dirac pulses, whereas the diffuse portion can be represented by an integrating-sphere model (an exponential 
function as discussed by Jungnickel et al., [14]). The channel frequency response, in terms of optical power, can then be written as

$$
H(f)=\sum_{i} \eta_{L O S, i} \exp \left(-j 2 \pi f \Delta \tau_{L O S, i}\right)+\eta_{D I F F} \frac{\exp \left(-j 2 \pi f \Delta \tau_{\text {DIFF }}\right)}{1+j f / f_{0}},
$$

where $\eta_{L O S}$ and $\eta_{\text {DIFF }}$ represent the channel gain for the LOS and diffuse signal respectively; $\Delta \tau_{\text {LOS }}[\mathrm{s}]$ and $\Delta \tau_{\text {DIFF }}[\mathrm{s}]$ are the corresponding signal delays, and $f_{0}[\mathrm{~Hz}]$ the cut-off (3$\mathrm{dB}$ ) frequency of the purely diffuse channel. The LOS gain from the $i^{\text {th }}$ LED chip is given by

$$
\eta_{L O S, i}=A_{R}(m+1) \cos ^{m} \Phi_{i} \cos \psi_{i} /\left(2 \pi r_{i}^{2}\right),
$$

where $A_{R}\left[\mathrm{~m}^{2}\right]$ is the effective receiver surface (together with filter and concentrator gain), and the other variables are introduced in Fig.1. For the purpose of easy comparison, we follow the work of Komine et al. [4] and assume a Si PIN photodiode with $1 \mathrm{~cm}^{2}$ surface and a concentrator with gain $3\left(120^{\circ}\right.$ FOV), together with a blue spectral filter without pass-band attenuation, summing up to an effective surface of $A_{R}=3 \mathrm{~cm}^{2}$. This is realistic with available technology (see, e.g. [15]). Nevertheless, in contrast to [4], where the optical filter passband covers the whole white-light region, we are detecting only blue light, and can therefore use a filter of much narrower spectral width $(\sim 130 \mathrm{~nm})$, and thus considerably reduce the influence of ambient light. According to the used model, the diffuse signal gain is constant everywhere in the room, and depends only on $A_{\mathrm{R}}$ and the room properties (room area $A_{R O O M}\left[\mathrm{~m}^{2}\right]$ and average reflectivity, $\rho$ )

$$
\eta_{\text {DIFF }}=\frac{A_{R}}{A_{\text {ROOM }}} \frac{\rho}{1-\rho} .
$$

The cut-off frequency, $f_{0}$ depends on the room properties, and is about $10 \mathrm{MHz}$ for a medium-sized room [14]. Nevertheless, as will be shown below, the diffusive component has no significant influence on the overall channel bandwidth.

Inter-symbol interference (ISI) stems from multi-path propagation of the emitted signals (through cables as well as air), and limits the transmission speed. The amount of ISI depends on the chosen transmission scenario (room properties, distribution of emitter chips at the ceiling and chip properties itself). We assume that for each submitted symbol all signals arriving at the receiver with a delay of more than half of the symbol period after the first signal contribute to ISI. Therefore, the received optical signal power is

$$
P_{R, \text { sig }}=\sum_{i} P_{R}\left(t_{i} \leq T_{s} / 2\right) \text { and } P_{R, I S I}=\sum_{i} P_{R}\left(t_{i}>T_{s} / 2\right) \text {. }
$$

Given the bandwidth of the transmitted signal is limited to $20 \mathrm{MHz}$ (LED modulation bandwidth), the Nyquist symbol period is limited to $25 \mathrm{~ns}$, and ISI will occur if transmitted data symbols experience delays larger than $12.5 \mathrm{~ns}$. In a more realistic case, when raised-cosine pulses with a roll-off factor of e.g., $\beta=0.3$, ISI will occur when delays are larger than $18 \mathrm{~ns}$.

We investigated the possibility for ISI in the introduced scenarios. The maximal delay between two optical LOS paths was determined by the receiver FOV, and the distance between transmitter and the desk-top surface. In our case, this delay is $\Delta t_{\text {air }} \leq 5.5 \mathrm{~ns}$, so ISI stemming from different optical LOS paths alone is not present. Nevertheless, this is the case when all LEDs are synchronously driven. A large difference in electrical signal paths from a common distribution point to each of the LED chips can also lead to ISI. Minimal difference in cable length, between two chips for which one can expect ISI to occur, is given by

$$
\Delta s \geq 0.75 c\left(T_{s} / 2-\Delta t_{\text {air }}\right),
$$

where $0.75 c$ is assumed for the speed of signals through cable. Assuming the maximal delay through air, this critical cabling difference comes to $2.8 \mathrm{~m}$ in the case with raised cosine shaping ( $1.6 \mathrm{~m}$ with the Nyquist symbol period). If this path difference is exceeded, ISI occurs and one can expect major influence on achievable transmission rates. Therefore, it is of interest to set the maximal electrical path difference as a requirement on cabling design. This tolerance of few meters should be quite comfortable to fulfill by implementation, especially in scenario B. For example, a convenient distribution point could be in the center of the ceiling, leading to the four lamps. Hereby, it is realistic to assume that the chips within one lamp are simultaneously driven. In the scenario A, the appropriate cabling would be more challenging. This is one of the reasons why scenario B is more realistic than $A$, apart from the fact that it requires the lower amount of LEDs. Our further investigations will therefore be based primarily on the scenario $\mathrm{B}$.

To determine the influence of signals coming via diffuse reflections, we obtained the $3-\mathrm{dB}$ frequency of the total channel at the desk-top surface, according to

$$
\left|H\left(f_{3 d B}\right)\right|^{2}=0.5|H(0)|^{2}
$$

and (6).

Figure 6 shows the distribution of $3-\mathrm{dB}$ frequencies for both scenarios. It can be observed that the $f_{3 d B}$ pattern somewhat reflects the ceiling design for both scenarios (i.e., the available bandwidth in scenario $\mathrm{A}$ is quite uniform, whereas for scenario B much larger variation of bandwidth is observable due to concentrated distribution of sources). Moreover, it can be concluded that the given bandwidth patterns are result of destructive as well as constructive interference of LOS signal phases stemming from different chips (i.e. lamps). In both scenarios, spans of available bandwidth are well above $20 \mathrm{MHz}$ (with minimal frequencies of $90 \mathrm{MHz}$ at the worst location at the desk-top surface, coincidentally for both scenarios A and B). Thus, the channel can be considered flat over the bandwidth of interest, and the influence of ISI can be neglected. With appropriate cabling, all LOS signal 
components arrive at the receiver within less than half of the symbol time and the influence of diffuse reflections is suppressed by strong LOS light all over the room. Nevertheless, even with $T_{s}=25 \mathrm{~ns}$, ISI can potentially be encountered in large rooms with high ceilings (e.g., conference halls) where differences in optical paths become significant with respect to the symbol length.
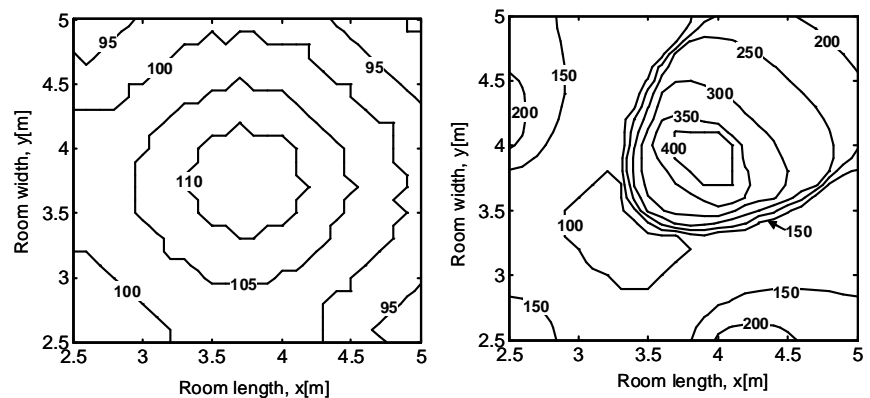

Fig. 6: 3-dB cut-off frequency (bandwidth) $[\mathrm{MHz}]$ distribution for the scenarios A (left) and B (right).

Note that, even though the channel is flat over $20 \mathrm{MHz}$, the LED modulation response at this frequency will be about $3 \mathrm{~dB}$ below the maximum and will therefore determine the frequency response in the realistic system. For the sake of simplicity, we have neglected this effect in the investigations in this paper, which leads to a slight overestimation of results. In a subcarrier-based system, this issue would be counteracted by e.g., pre-emphasizing the transmission power spectrum at higher frequencies [1] or allocating lower modulation orders to them [16], [17].

\section{B. System Performance and Achievable Transmission Rate}

We assume that all LEDs are driven by the same (electrical) signal. Since the tolerance for the electrical path difference between the lamps in scenario B is relatively large, we assume that cabling is within this tolerance. Then, in a flat channel, the received optical signal power is the sum of powers coming from all light emitting chips

$$
P_{R}=\sum_{i}\left|H_{i}(0)\right| P_{T, i}=P_{\text {CHIP }} \sum_{i} \eta_{L O S, i}=P_{C H I P}|H| .
$$

For simulation it is easier to regard it as one source, $P_{C H I P}$, and one virtual channel, $|H|$, which is the sum of all LOS gains. We define a reference signal-to-noise ratio as the total electrical signal power generated by the photodiode (containing the DC component) over the AWGN power in bandwidth $B$ (20 MHz)

$$
S N R_{0}=2 \gamma^{2} P_{R}^{2} /\left(N_{0} B\right)=2 \gamma^{2} P_{C H I P}^{2}|H|^{2} /\left(N_{0} B\right),
$$

where $\gamma[\mathrm{A} / \mathrm{W}]$ denotes the receiver responsivity, and $N_{0}$ the double-sided power spectral density of the noise $\left[\mathrm{A}^{2} / \mathrm{Hz}\right]$. The responsivity of Si-based photodiodes is $\sim 0.28 \mathrm{~A} / \mathrm{W}$ in the blue region. The dominant noise contribution is assumed to be the shot noise stemming from ambient light from windows. As a sort of a worst case, bright sky irradiance of $5800 \mu \mathrm{W} /\left(\mathrm{nm} \cdot \mathrm{cm}^{2}\right)$ was considered [18]. After blue-light filtering, the induced photocurrent at the assumed receiver is $I_{a l}=0.62 \mathrm{~mA}$ (about four times smaller than assumed in [4]), and the noise power spectral density is

$$
N_{0} \cong N_{\text {shot }}=q I_{a l} \sim 10^{-22} \mathrm{~A}^{2} / \mathrm{Hz},
$$

where $q$ is the electron charge.

Figure $7 \mathrm{a}$ presents the $S N R_{0}$ distribution at the desk-top surface for scenario $\mathrm{B}$ in the case where cabling design is within tolerance, and the values are summarised in Table 1.
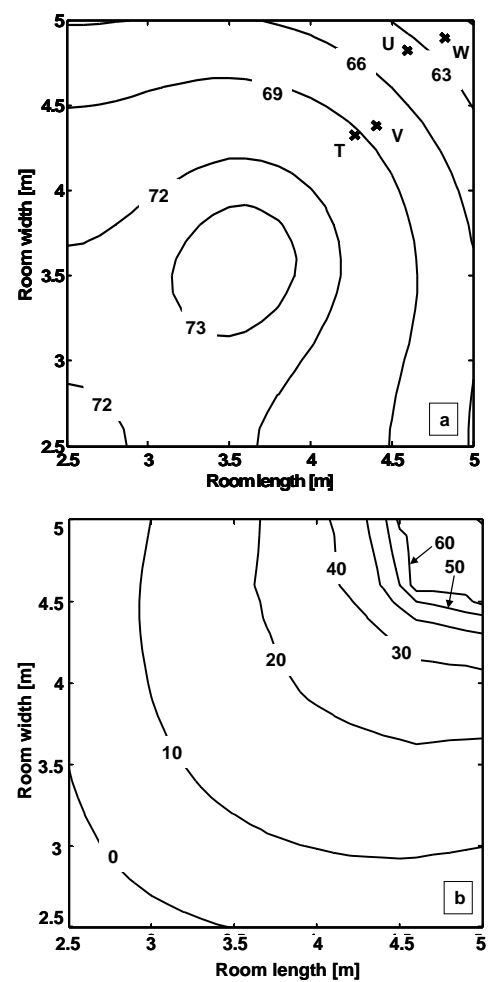

Fig. 7: Distribution of the reference SNR $\left(S N R_{0}[\mathrm{~dB}]\right)$ on the working plane for the scenario B (a) with appropriate cabling, (b) with unfortunate cabling. The crosses stand for the receiver positions for which simulations are performed later on.

Since the SNR is not affected by ISI and the illuminance level is comparably high, the $S N R_{0}$ remains above $60 \mathrm{~dB}$ over the whole plane. After simple mathematical transformations, the $S N R_{0}$ can be expressed as a function of the brightness level, E:

$$
S N R_{0}=2 E^{2}\left(\gamma A_{R} \alpha\right)^{2} /\left(N_{0} B\right) .
$$

For $400 \mathrm{~lx}, S N R_{0}$ is slightly above $66.6 \mathrm{~dB}$, making this the minimal $S N R_{0}$ on the recommended working plane (covering $\sim 87 \%$ of the desk-top surface for scenario B).

Just for comparison purposes, $S N R_{0}$ distribution was also calculated with the worst-case cabling scenario, when the signals arriving to the receiver from three out of four lamps 
cause ISI, and presented in Fig. $7 \mathrm{~b}$. In this case $S N R_{0}$ is given as

$$
S N R_{0}=2 \gamma^{2} P_{R, \text { sig }}^{2} /\left(N_{0} B+\gamma^{2} P_{R, I S I}^{2}\right) .
$$

It can be seen that difference in electrical path lengths can cause significant reduction of $S N R_{0}$ and consequently limit the transmission rate. Clearly, the drop is greatest towards the centre of the working plane, where the three lamps causing ISI have the greatest influence. Therefore, awareness by cabling design is of great interest.

In our system, the signal bandwidth is limited to $20 \mathrm{MHz}$ by the modulation bandwidth of the LED. If raised-cosine pulses with a roll-off factor of $\beta=0.3$ are assumed, bit rates much higher than $30 \mathrm{Mbit} / \mathrm{s}$ can only be achieved if bandwidth efficient modulation formats are used. Due to intensity modulation/direct detection (IM/DD) type of optical wireless systems, pulse-amplitude modulation with $M$ levels ( $M$-PAM) is a bandwidth-efficient modulation format for base-band transmission. It is, however, well known from electrical communication theory that, for the same BER, QAM requires a lower SNR than PAM (also than PSK) assuming the same order of modulation $M$ [19], but this type of modulation is only possible in our system if sub-carrier modulation is used. In the following, we evaluate and compare the performances of base-band transmission (using $M$-PAM) and sub-carrier transmission (based on DMT [19], [20], in combination with $M$-QAM).

Figure 7a shows that the SNR can exceed as high levels as $70 \mathrm{~dB}$. For such high SNR values the random optical beating of the optical power emitted by the incoherent white-light LEDs becomes noticeable. According to the literature, LEDs are to a good approximation thermal emitters [21] and when neglecting all other sources of random variation, the achievable SNR for un-polarised thermal light is given by

$$
S N R_{\text {beat }}=2 \Delta v / \Delta f,
$$

where $\Delta v$ is the optical bandwidth of the detected light and $\Delta f$ is the bandwidth of the detection system. Notice, that this equation only holds for $\Delta v \square \Delta f$. A thorough discussion of the optical beating of (thermal) light can be found elsewhere [21], [22]. In our case the former is $\sim 125 \mathrm{THz}$. Assuming the measurement bandwidth to be around $40 \mathrm{MHz}$, equation (15) yields an SNR of $69 \mathrm{~dB}$, i.e., throughout the whole area for which the luminous flux is above $400 \mathrm{~lx}$ the achievable SNR is capped to about $69 \mathrm{~dB}$.

\section{1) M-PAM based transmission}

An $M$-PAM transmission scheme is shown in Fig. 8. The LED is modulated by a stream of $M$-PAM symbols,

$$
x(t)=\left(A_{k}+A_{\text {CHIP }}\right) g\left(t-k T_{\text {sym }}\right),
$$

where $A_{\text {CHIP }}$ represents the mean signal amplitude, $A_{k}$ the scaled amplitude of the $k^{\text {th }}$ symbol interval of length $T_{\text {sym }}$, and $g(t)$ the pulse shape.

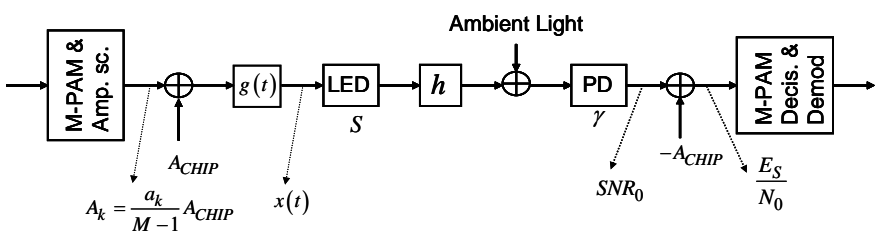

Fig. 8: Block scheme for an M-PAM transmission chain.

Namely, the $M$-level symbol alphabet has $M$ different values $a_{k} /(M-1), \quad$ where $\quad a_{k} \in\{-(M-1), \ldots,-1,1, \ldots,(M-1)\}$, which are occurring with equal probabilities. To properly drive the optical source (i.e., to ensure the mean optical power of $\left.P_{C H I P}\right)$ symbol amplitudes need to be scaled with $A_{\text {CHIP }}=P_{\text {CHIP }} / S$, ( $S$ being the LED conversion factor in [W/A]) so that $A_{k}=\frac{a_{k}}{M-1} A_{C H I P}$, and a DC of $A_{C H I P}$ needs to be included. The SNR is then given as

$$
\begin{aligned}
\frac{E_{S}}{N_{0}} & =\frac{S^{2}\left\langle A_{k}^{2}\right\rangle|H|^{2} \gamma^{2}}{N_{0} B}=\frac{\left\langle a_{k}^{2}\right\rangle P_{C H I P}^{2}|H|^{2} \gamma^{2}}{(M-1)^{2} N_{0} B} \\
& =\frac{M+1}{3(M-1)} \frac{P_{C H I P}^{2}|H|^{2} \gamma^{2}}{N_{0} B}=f_{P A M}(M) \frac{S N R_{0}}{2} .
\end{aligned}
$$

Given that the factor $f_{P A M}(M)$ presents a monotonic decreasing function of $M$, which approaches $1 / 3$ for $M \rightarrow \infty$, it is clear that for higher $M$ more power is needed for the same SNR, i.e., the power-efficiency decreases. This factor actually represents the ratio between the mean and peak electrical power.

According to [19], the relationship between BER and $E_{S} / N_{0}$ for 20-MBd $M$-PAM signals is

$$
\begin{aligned}
B E R_{M-P A M} & \cong \frac{M-1}{M} \frac{2}{\log _{2} M} Q\left(\sqrt{\frac{3}{M^{2}-1} \frac{E_{S}}{N_{0}}}\right) \\
& \cong \frac{M-1}{M} \frac{2}{\log _{2} M} Q\left(\frac{\sqrt{S N R_{0} / 2}}{M-1}\right) .
\end{aligned}
$$

The approximation sign is here introduced since with Gray coding, each symbol error "only" most likely causes one bit error.

Now, for a chosen bit-error performance and taking into account the $S N R_{0}$ span present in the considered scenario, we can determine the maximal allowed order of $M$-PAM at the desk-top surface. Then, the achievable transmission rate is given by 


$$
R=\frac{2}{1+\beta} B \log _{2} M
$$

This is illustrated in Fig. 9, where rates as high as $300 \mathrm{Mbit} / \mathrm{s}$ can be achieved for BER $=10^{-3}$.

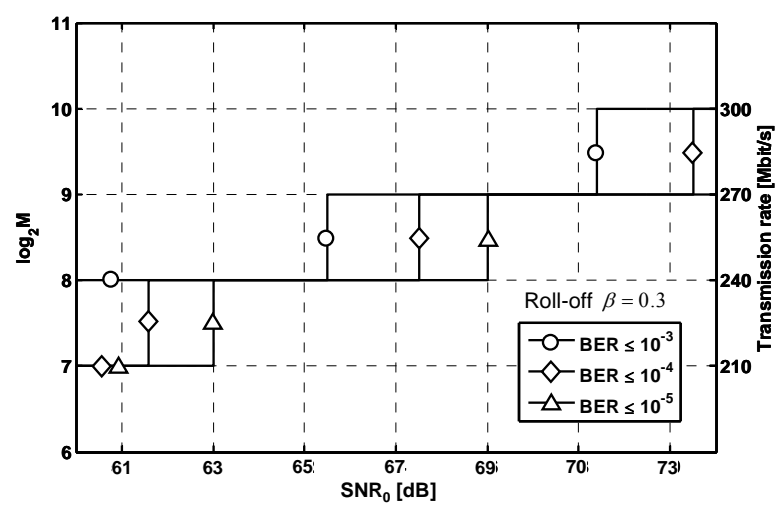

Fig. 9: $M$-PAM order of modulation (left ordinate) and transmission rate (right ordinate) for various bit-error ratios and as function of the base-band SNR.

To support the above conclusions, Monte Carlo simulations have been performed in points $\mathbf{T}$ and $\mathbf{U}$ (shown in Fig. $7 \mathrm{a})$, and the BERs obtained from simulations were compared to the BERs rendered with (18). In simulations, perfect synchronization and decision thresholds are assumed. Since the available SNRs at the receiver plane are extremely high, the Monte Carlo simulations with given parameters would consider very high modulation orders or very low BERs, which in turn would result in a prohibitively large number of calculation steps. Therefore, the simulations in points $\mathbf{T}$ and $\mathbf{U}$ were performed assuming LED chips of ten times smaller optical power (leading to a $20 \mathrm{~dB}$ lower SNR). The BERs inferred from theory and simulations were found to differ less than $5 \%$. This discrepancy is a consequence of the fact that the number of simulated symbol errors is limited by computation complexity to about 100 . However, such difference is well in the range of a $90 \%$ confidence interval.

\section{2) DMT transmission}

In a fading channel, sub-carrier modulation enhances system robustness. In an optical wireless system on the other hand, moving the signal spectrum away from DC offers better robustness against ambient-light noise and interference form fluorescent lighting, which is particularly strong around DC.

In particular, DMT is a special type of modulation technique that offers high bandwidth efficiency, inherently deals with ISI, allows for simple equalization at the receiver, and can be entirely realised with digital signal processing [19], [20].

As shown in Fig. 10, at the Tx the input data are divided into several parallel streams, which will be encoded and imprinted onto different sub-carriers. These are then mapped to $M$-QAM symbols by aid of Gray coding. A DMT symbol is formed via digital signal processing, and used to directly modulate the optical source. At the input of the Inverse Fast Fourier Transformation (IFFT) block, conjugate symmetry is enforced on the vector of QAM symbols, to ensure a realvalued modulation signal. This means that for $\mathrm{N}-1$ independent sub-carriers (first input of the IFFT block corresponds to DC, which does not carry any data), a $2 N$-IFFT block is necessary.

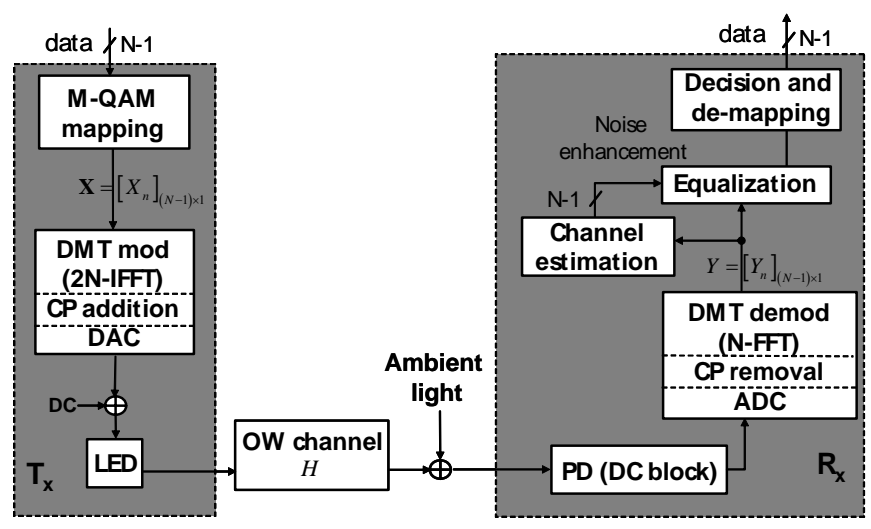

Fig. 10. Block scheme of a DMT transmission chain with M-QAM mapping.

After detection and DMT demodulation, sub-carriers are separately processed. Following the channel estimation, the data are equalized (e.g., multiplied with an inverse channel coefficient).

The SNR on each subcarrier is

$$
\frac{E_{S}}{N_{0}}=\frac{S^{2}\left\langle\left|X_{n}\right|^{2}\right\rangle(\gamma|H|)^{2}}{2 N_{0} B_{n}},
$$

where $2 N_{0} B_{n}$ is the noise power on one sub-carrier. For a system with $N$ independent sub-carriers and $M$-QAM mapping, the SNR of each sub-carrier will be

$$
\begin{aligned}
\frac{E_{S}}{N_{0}} & =\left(\frac{P_{C H I P}}{N-1}\right)^{2} f_{D M T}(M) \frac{(\gamma|H|)^{2}}{2 B N_{0} / 2 N} . \\
& =\frac{N}{2(N-1)^{2}} f_{D M T}(M) S N R_{0}
\end{aligned} .
$$

The SNR is a function of the number of sub-carriers and the receiver position.

Here, the factor $f_{D M T}(M)$ corresponds to the ratio between the mean electrical power (i.e. mean of the square signal amplitude) and the square of the mean optical power (i.e. mean signal amplitude)

$$
f_{D M T}(M)=\frac{\left\langle|X|^{2}\right\rangle}{\langle|X|\rangle^{2}}=\frac{\left\langle|X|^{2}\right\rangle}{\left(\frac{P_{C H I P}}{N-1}\right)^{2}} .
$$

Such approach will lead to a certain amount of clipping noise, especially for low numbers of sub-carriers. The optimization of the clipping level is currently under investigation. In 
order to ensure negligible clipping at the optical source, it was assumed that the sum of the mean signal amplitudes on individual subcarriers is $P_{C H I P} / S$. According to [19] the theoretical formula connecting BER and $E_{S} / N_{0}$ for $M$-QAM is

$$
B E R \cong \frac{4}{\log _{2} M} \frac{\sqrt{M}-1}{\sqrt{M}} Q\left(\sqrt{\frac{3}{M-1} \frac{E_{S}}{N_{0}}}\right),
$$

where the approximation is valid for high SNRs and Gray coding. By introducing (23) into (25), the BER dependence on $S N R_{0}$ can be found as

$$
B E R \cong \frac{4}{\log _{2} M} \frac{\sqrt{M}-1}{\sqrt{M}} Q\left(\frac{\sqrt{N}}{N-1} \frac{\sqrt{S N R_{0}}}{\left\langle A_{s}(M)\right\rangle}\right),
$$

where $N$ is the number of used sub-carriers and $\left\langle A_{s}(M)\right\rangle$ is the mean amplitude of the symbol alphabet. For square $M$ QAM constellations

$$
\left\langle A_{s}(M)\right\rangle=\frac{4}{M} \sum_{a=1,3, \ldots}^{\sqrt{M}-1} \sum_{b=1,3, \ldots}^{\sqrt{M}-1} \sqrt{a^{2}+b^{2}}
$$

can be easily derived.

Again, we can determine the maximal order of $M$-QAM modulation according to the chosen system bit-error performance and $S N R_{0}$ span present in the considered scenario. The corresponding net transmission rate will also depend on the assumed length of the cyclic prefix $L$, i.e.,

$$
R=\frac{2(N-1)}{2 N+L} B \log _{2} M
$$

In Fig. 11 the maximal number of bits per sub-carrier are given for the cases $N=8$ (with $L=1$ ) and $N=16$ (with $L=2$ ), as well as the corresponding transmission rates (right ordinate).

We also conducted simulations for the DMT system in points $\mathbf{V}$ and $\mathbf{W}$ as depicted in Fig. 7a, and also found them to deviate less than $5 \%$ from the predictions in Fig. 11 . The chosen cyclic prefix lengths are sufficient, since the channel memory (calculated to be up to $5.5 \mathrm{~ns}$ in the considered scenario) is well below the sampling interval of $25 \mathrm{~ns}$.

When comparing Fig. 11 and Fig. 9 the achievable rates with DMT and PAM are quite within the same range. The decision between the two approaches would thus have to be made depending on the environment (noise and ISI) and desired system complexity. Even though QAM combined with DMT is more complex than PAM, it provides a slightly higher maximum data rate for the same optical SNR and it is more robust regarding noise. In practice, one should also aim towards a flexible system that is robust in ISI-degraded environments, e.g. large lecture halls. ISI degrades the channel response on certain frequencies. In the case of base-band transmission, performance in the whole channel is corrupted (without any equalization), so the resulting SNR can be significantly lower. For instance, in a scenario where $10 \%$ of the total received power is delayed enough to contribute to ISI, it can be shown that the SNR will be in the range of about $20 \mathrm{~dB}$, (since instead of ambient light now the delayed signals present the major interference), allowing for, e.g., only $60 \mathrm{Mbit} / \mathrm{s}$ with 4-PAM $\left(\beta=0.3, \mathrm{BER}=10^{-4}\right)$. In this case, DMT allows for much better performance close to the values shown in Fig. 11. In the case that the degradation involves only some sporadic frequencies, only some of the sub-carriers would suffer performance degradation, whereas the rest of them would still have high SNRs. If the response is degraded on a whole frequency band, then one could deploy adaptive DMT, i.e. modulating the sub-carriers with different QAM orders depending on the SNR of each one [16], [17].
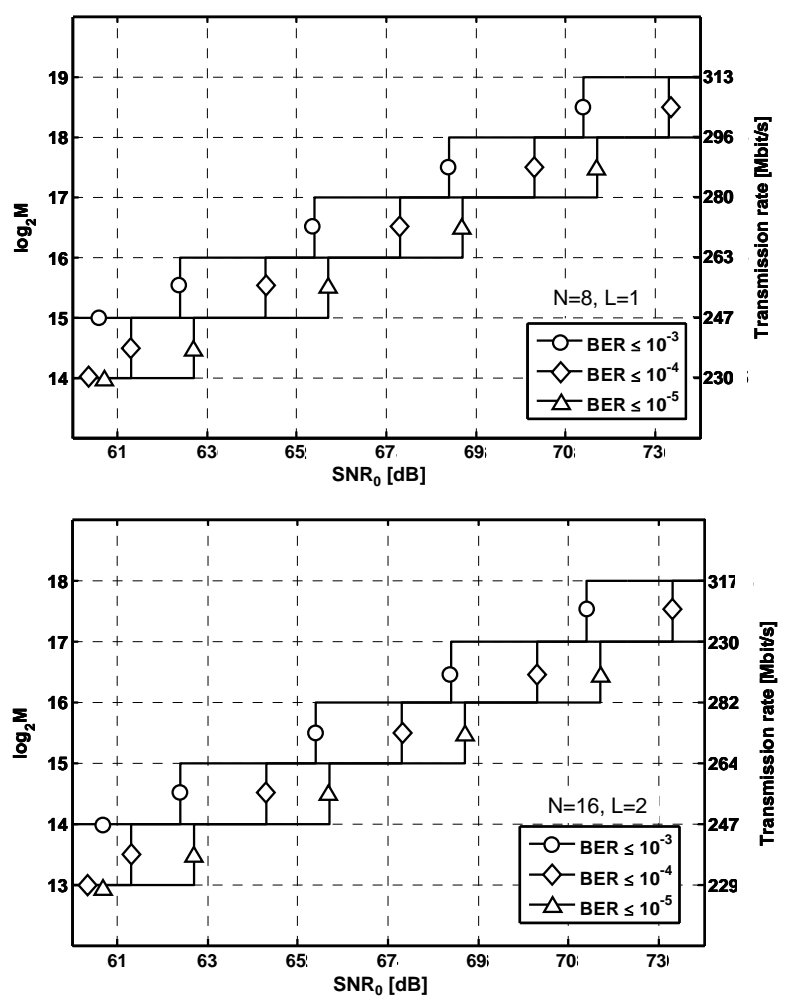

Fig. 11: $M$-QAM order of modulation (left ordinate) and achievable transmission rates (right ordinate) for $N=8$ (top) and $N=16$ (bottom) DMT sub-carriers for various bit-error rates and as a function of base-band SNR with cyclic prefix $L=1$ and $L=2$, respectively.

\section{CONCLUSIONS}

Using white-light LEDs simultaneously for illumination and data transmission promises synergy, which is increasingly gaining attention in $R \& D$ [23]. We have shown that the modulation bandwidth of commercially available LEDs can be enhanced to $\sim 20 \mathrm{MHz}$ when suppressing the phosphorescent portion of the optical spectrum upon detection. Starting from requirements for office illumination, as defined in lighting standards, we investigated achievable transmission rates in a medium-sized room model. Assuming that the cabling is done within what was found to be a comfortable tolerance, the 
transmission channel can be regarded flat over the bandwidth of interest, due to high optical power distributed via many line-of-sight links. The system performances were compared for base-band $M$-PAM and $M$-QAM on DMT modulation. It was found that the achievable rates are comparable for both techniques and lie in the region of several hundreds Mbit/s. It can be expected that benefits of DMT are even pronounced in the ISI degraded environment. Analytical assumptions were corroborated with Monte Carlo simulations for both modulation schemes. Even though a DMT system is more complex, it can be fully realized with standard FPGA and DSP units. In practice, we expect the reported large SNR values to be limited by the linearity of the transmitter and receiver, and experimental investigations are necessary to fully assess the practical potential of high-speed white-light wireless transmission.

\section{REFERENCES}

[1] J. Grubor, S.C.J. Lee, K.-D. Langer, T. Koonen, J.W. Walewski, "Wireless high-speed data transmission with phosphorescent white-light LEDs," in Proc. ECOC 2007, vol. 6, PD3.6.

[2] J.-H. Kim, et al., "Visible light communication at $20 \mathrm{Mbit} / \mathrm{s}$ using illumination LEDs," in Proc. of SPIE, vol. 6353, 2006.

[3] Y. Tanaka, T. Komine, S. Haruyama and M. Nakagawa, "Indoor visible communication utilizing plural white-light LEDs as lighting," in Proc. PIMRC 2001, pp. F81-F85.

[4] T. Komine and M. Nakagawa, "Fundamental analysis for visible-light communication system using LED lightings," IEEE Trans. on Consumer Electronics, vol. 50, no. 1, pp. 100-107, 2004

[5] P. Amirshahi and M. Kavehrad, "Broadband access over medium and low voltage power-lines and use of white-light light emitting diodes for indoor communications," in Proc. IEEE CCNC 2006, pp. 897-901.

[6] S.-B. Park et al., "Information broadcasting system based on visible light signboard," in Proc. Wireless and Optical Communications 2007, pp. 311-313.

[7] G. Pang, T. Kwan, H. Liu and C.-H. Chan, "LED wireless - A novel use of LEDs to transmit audio and digital signals," IEEE Industry Applications Magazine, vol. 8, no. 1, pp. 21-28, 2002.

[8] S. Kitano, S. Haruyama M. Nakagawa, "LED road illumination communications system," in Proc. VTC-Fall, 2003, pp. 3346-3350.

[9] T. Komine, S. Haruyama and M. Nakagawa, "Performance evaluation of Narrowband OFDM on integrated system of power line communication and visible light communication," in Proc. IEEE Symp. on Wireless Pervasive Computing, 2006.

[10] M.Z. Afgani, H. Haas, H. Elgala and D. Knipp, "Visible light communication using OFDM," in Proc. 2nd International IEEE/Create-Net Conf. on Testbeds and Research Infrastructures for the Development of Networks and Communities, 2006.

[11] www.osram.de (DOT-it).

[12] E.F. Schubert, "Light-emitting diodes," Cambridge University Press, 2003.

[13] European standard EN 12464-1: Lighting of indoor work places, 2003.

[14] V. Jungnickel, V. Pohl, S. Noenning and C. von Helmolt, "A physical model for the wireless infrared communication channel," IEEE JSAC vol. 20, no. 3, pp. 631-640, 2002.

[15] http://sales.hamamatsu.com (Si PIN photodiode S6968 series), 2006.

[16] S. Randel, et al., "1 Gbit/s transmission with $6.3 \mathrm{bit} / \mathrm{s} / \mathrm{Hz}$ spectral efficiency in a $100 \mathrm{~m}$ standard $1 \mathrm{~mm}$ step-index plastic optical fibre link using adaptive multiple sub-carrier modulation," in Proc. ECOC 2006, vol. 6 , pp. 41-42.

[17] J. Grubor, V. Jungnickel and K.-D. Langer, "Adaptive-modulation technique in wireless infrared indoor communication," in ITC Fachbericht 193, pp. 193-200, 2006.

[18] J.R. Barry, "Wireless infrared communications," Kluwer Academic Publishers, Boston, Dordrecht, London, 1994.

[19] F. Xiong, "Digital modulation techniques," Artech House, Boston, London, 2000.

[20] J.M. Cioffi, “A multicarrier primer," ANSI T1E1, 1991.
[21] J.W. Walewski, J.A. Filipa and S. T. Sanders, "Optical beating of polychromatic light and its impact on time-resolved spectroscopy. Part I: Theory," Applied Spectroscopy, vol. 62, no. 2, pp. 9-18, 2008.

[22] J.W. Goodman, "Statistical optics," John Wiley \& Sons, Inc., New York, 1985.

[23] https://mentor.iee.org/802.15/file/08/15-08-0214-01-0vlc-ig-vlcclosing-report.ppt

Jelena Grubor obtained diploma degree in Electrical Engineering with emphasis on Telecommunications, at Belgrade University in 2002. In 2003, she joined the Fraunhofer Institute for Telecommunications, Heinrich-HertzInstitut, Berlin as a $\mathrm{PhD}$ student, where she works on access and indoor networks with focus on optical wireless communication.

Sebastian Randel (S'02-M'06) received his Dipl.-Ing. as well as his Dr.-Ing. degree from Technical University Berlin in 2001 and 2005, respectively. His dissertation work dealt with physical layer issues of WDM transmission systems operating at $160 \mathrm{Gbit} / \mathrm{s}$ per wavelength channel. In 2005 he joined Siemens AG, Corporate Technology in Munich as a research scientist. Currently, he is involved in research activities on advanced Ethernet PHY for polymer optical fiber and optical wireless communications. His further research interests include cost-efficient PON architectures, as well as advanced modulation formats for 100-Gbit/s transport networks.

Klaus-Dieter Langer graduated at Braunschweig-Wolfenbuettel University of Applied Sciences and received the diploma degree (Dipl.-Ing.) in Electrical Engineering from the Technical University of Berlin, Germany. In 1981, he joined Heinrich-Hertz-Institut (HHI), Berlin, as a research associate working on broadband communications. He has been involved in numerous research projects where he has worked mainly on the design and performance evaluation of photonic switching systems and networks. In 1992, he changed to the German Federal Ministry of Research and Technology where he served as an adviser on national telecommunications, digital audio broadcasting, and photonics/ optoelectronics R\&D. Subsequently at the HHI, he addressed the employment of optical and photonic technologies in communication networks, their layout and in particular the topic of cost-efficient optical subscriber lines and the use of WDM for optical access. Currently he is heading the research on optical access and indoor networks at the Photonic Networks and Systems Department of the Fraunhofer Institute for Telecommunications, HHI.

Joachim W. Walewski graduated in 1995 from the Christian Albrechts University, Germany, with a diploma degree in physics (Dipl.-Phys.) and he earned a PhD from the Lund Institute of Technology, Sweden, in 2002 for his research in the field of applied laser spectroscopy. From 1996 to 1997, he was a visiting scientist at the Tampere University of Technology, Finland, engaging in laser-assisted diagnostics of CVD diamond. From 2001 to 2003, he served as junior lecturer at the Lund Institute of Technology, and from 2003 to 2006 he held positions as research associate and finally as assistant scientist at the Engine Research Center of the University of Wisconsin Madison, U.S.A. At the latter two institutions, his research was focused on the development and application of laser-spectroscopic techniques for combustion research. In May 2006 he joined Siemens Corporate Technology, Information \& Communications, in Munich, Germany and has focused his efforts on R\&D in the field of wireless optical communications. 